\title{
Validation of a Feedback-Controlled Elbow Simulator: Elbow Muscle Moment Arm Measurement
}

\author{
Laurel Kuxhaus $^{1,2}$, Patrick J. Schimoler ${ }^{2,3}$, Jeffrey S. Vipperman ${ }^{3,1}$, Mark Carl Miller ${ }^{2,3}$ \\ 5 \\ ${ }^{1}$ Department of Bioengineering, University of Pittsburgh, Pittsburgh, PA \\ ${ }^{2}$ Orthopaedic Biomechanics Research Laboratory, Allegheny General Hospital, \\ Pittsburgh, PA \\ ${ }^{3}$ Department of Mechanical Engineering \& Materials Science, University of Pittsburgh, \\ Pittsburgh, PA
}

Corresponding author:

Mark Carl Miller, Director

20 Orthopaedic Biomechanics Research Laboratory 320 E. North Ave. (AGH)

$10^{\text {th }}$ Floor, South Tower

Pittsburgh, PA 15212

412.359.5282 (phone)

25412.359 .8682 (fax)

mcmiller@wpahs.org

Word Count: 3582

Key words:

35 Elbow

Joint simulator

Closed-loop control

Muscle moment arms

Physiologic simulator design 


\begin{abstract}
:
The AGH Elbow Simulator was designed to be a physiologic simulator actuating motion via the elbow's native musculotendons. As such, it must offer physiologically correct adjustable moment arms throughout the elbow's range of motion. The system is

5 described here and an essential component of its design is validated. A salient feature of the physical design is a robustly designed frame that can be rotated to test elbows with both varus and valgus loads. The simulator produces movement in cadaveric elbow specimens via servoelectric actuators that attach to the tendons of the biceps, brachialis, triceps, and pronator teres muscles. Force and position control is possible. Actuator

10 position, elbow position, and musculotendon forces can be used in closed-loop feedbackcontrol algorithms. The frame and actuators were chosen to be able to replicate all speeds of elbow movement, including the fast movements necessary to perform throwing activities such as baseball pitching.
\end{abstract}

15 The Simulator was designed to maintain physiologically accurate moment arms throughout the elbow's range of motion. To validate this design goal, muscle moment arms were measured in three cadaver elbow specimens using the Simulator. Flexionextension moment arms of four muscles were measured at three different pronation/supination angles: fully pronated, fully supinated, and neutral. Pronation-

20 supination moment arms were measured at three different flexion-extension angles: $30^{\circ}$, $60^{\circ}$, and $90^{\circ}$. The tendon-displacement method was used to compute the moment arms as the ratio of the change in musculotendon length to the change in joint angle. The numeric results compared well with those previously reported. The biceps and pronator 
teres flexion-extension moment arms varied with pronation-supination position, and vice versa. This represents the first use of closed-loop feedback control in the AGH Elbow Simulator, is one of the few reports of both flexion-extension and pronation-supination moment arms in the same specimens, and demonstrates the adjustability of the moment

5 arms that the elbow simulator can produce.

\section{Introduction:}

Complete elbow function is critical to daily life. Without full range of elbow motion $\left(30^{\circ}\right.$ to $130^{\circ}$ of flexion and $50^{\circ}$ of supination to $50^{\circ}$ of pronation), [6] activities of daily living

10 such as feeding and grooming are compromised and can decrease a person's functional independence. Elbow function can be impaired through both acute or chronic injury and trauma, such as radial head fractures, which account for $5.4 \%$ of all adult fractures. $[8,16,23]$

15 One approach to study human joints is to use a joint simulator. Human joint simulators using cadaveric specimens can be grouped into two categories: physiologic and kinetic. While kinetic simulators apply external forces to create joint movements, in a physiologic simulator, the control of the specimen comes internally from the physiologic structures. Forces are applied via tendons to actuate movement. The tendon-actuator interface with

20 the supporting structures clearly defines anatomic boundary conditions that recreate adjacent joint movements and muscle lines of actions. Muscle coactivations (in antagonist-agonist sets) can be included. The joint can then perform movements that cannot be distinguished from the movements of live subjects. Researchers have 
successfully used physiologic joint simulators in the wrist, elbow, shoulder, hip, knee and ankle. $[9,17,22,29,30]$

Physiologic simulators are challenging to control because the joints of the human body

5 have redundant actuators. That is, multiple muscle combinations can act together to create the same effect at each joint. This redundancy means that there can be infinitely many muscle activation combinations to produce a given movement or force at each joint. There is no commercially-available controller that replicates the human nervous system.

The only elbow joint simulator reported to date is a physiologic elbow simulator. [17] While this simulator has expanded the understanding of elbow reconstruction $[3,4,10,11,18,19]$, operates primarily in an open-loop manner; many iterations are required to create the desired arm motion; the arm is led through a prescribed flexion15 extension movement with a single fixed non-physiologic external moment, muscle synergism is based on static EMG data, muscle cross-sectional areas (CSA), and moment arms that are assumed to be fixed; and there is no antagonistic muscle control. The success of the Syracuse Wrist Simulator [30] with a straightforward PID control algorithm illustrates that feedback control of an upper-extremity joint simulator is

20 attainable and addresses many of the shortcomings of the above-described elbow simulator. In particular, since it uses feedback control, the desired motion can be achieved more quickly in a manner that is more representative of in vivo limb control. In regard of these reasons, a closed-loop feedback-controlled elbow simulator for use with 
cadaveric elbows has been developed. The AGH Elbow Simulator allows the cadaveric elbow specimen to achieve full range of motion in both flexion-extension $\left(30^{\circ}\right.$ to $130^{\circ}$ of flexion) and pronation-supination ( $50^{\circ}$ pronation to $50^{\circ}$ supination). [24] The entire frame can be placed on its side to permit specimen testing in orientations with varus or 5 valgus loads.

In order to successfully create physiologic elbow motion, the moment arms of each muscle about the elbow must be accurately reproduced. Moment arm values about the flexion-extension (f/e) axis of the elbow, measured in physical models or in cadaveric

10 specimens, have been reported for the biceps, brachialis, triceps, brachioradialis, and pronator teres. $[1,14,20,25,26]$ Moment arms about the pronation-supination (p/s) axis have also been reported. $[5,13]$ One common technique to measure moment arms is the tendon-displacement method. In this method, tendons are loaded with a constant force and the joint of interest is passively moved. Tendon excursion and joint movements are

15 recorded which permits the calculation of moment arms. Given the design features of the simulator, the hypothesis was that measuring moment arms in a cadaveric specimen in our simulator using closed-loop force control would yield results within previously reported ranges. [5,21]

\section{Methods:}

$\underline{\text { Basic Design Features: }}$

The elbow simulator was designed to facilitate complete elbow motion controlled by the four major muscles that cross the elbow: the biceps brachii, triceps brachii, pronator 
teres, and brachialis. The rigid steel frame, shown in Fig. 1, holds four servoelectric actuators which create elbow joint motion when attached to the muscles of a cadaver specimen. The actuators (ET50 cylinders coupled to BE-series motors) are controlled by Gemini GV drives (Parker-Hannifin, Rohnert Park, CA). The actuators control cables

5 routed through alignment pulleys which are attached to the muscles of a cadaveric elbow specimen. The cables are high-strength $\left(80-\mathrm{lb}\right.$, Stren ${ }^{\circledR}$ Super Braid, Stren, Spirit Lake, IA). The pulleys can be physically adjusted to accurately represent muscle insertion locations across a variety of elbow specimen sizes. [28] To maintain physiologically accurate moment arms throughout the elbow's motion, custom pulley mounts were

10 designed for the pronator teres to accommodate the geometric change in musculotendon angle that occurs with normal elbow motions (see Fig. 1, inset). Specifically, a portion of the pulley assembly can rotate to provide a physiologically accurate line of travel throughout p/s. CPS-2038 zinc-plated steel pulleys (Sava Industries, Inc.) were used throughout. A clamp secures the humerus of the cadaver specimen to the frame and

15 aligns it horizontally (Fig. 1). The entire frame can be rotated $\pm 90^{\circ}$ to simulate movements under both a varus and a valgus load. The pulleys and actuator attachments are designed to accommodate both right and left elbow specimens with simple hardware adjustments.

20 A controller card (ACR8020, Parker-Hannifin, Rohnert Park, CA) permits multiple tasks to run simultaneously. Data acquisition is a part of the controller card and can monitor muscle forces via in-line load cells (Model 31, Honeywell Sensotec, Columbus, OH) attached to each cylinder. The ACR8020 can sample these at rates up to $2 \mathrm{kHz}$. 
Potentiometers (P1401a, Novotechnik, Southborough, MA) can be attached at both the elbow joint and the distal radial-ulnar joint to measure f/e and p/s position, respectively. Alternatively, an inclinometer (X3Q, US Digital, Vancouver, WA) can be mounted to the ulna and used to measure the f/e position. A schematic of the control system hardware 5 arrangement is shown in Fig. 2.

\section{Specimen Preparation:}

Each of three cadaveric elbow specimens was carefully dissected to reveal the four tendon insertions of interest. Two were right arms. The mean specimen age was 68.3

10 (S.D. 6.74) years and all three were from female donors. Krackow whip stitches sutured into each tendon created attachment points for the lines to the actuators. For the triceps muscle, a nylon strap was sutured to the tendon to prevent damage at high loads. The other anatomic structures of the specimen were left intact from the mid-humerus to the distal radial ulnar joint. An axis finder, modeled after Hollister et al. [15] was used to

15 locate the $\mathrm{f} / \mathrm{e}$ and $\mathrm{p} / \mathrm{s}$ axes of the elbow. To keep the potentiometers aligned with the anatomic axes of rotation, custom mounts (Fig. 3) rigidly attached the potentiometers to the specimen.

\section{Evaluation of custom pulley design:}

20 To evaluate the apparatus, experiments to measure the moment arms in a cadaveric elbow were conducted. A proportional-only controller was used on each muscle to maintain constant force. Each tendon was loaded to a minimum of 13.34 N. The elbow was passively moved through six different motions while the cylinders adjusted their 
positions to maintain constant force on each muscle. The six motions were: f/e motion with $\mathrm{p} / \mathrm{s}$ angle held constant at 1) neutral, 2) fully pronated, 3) fully supinated; and $\mathrm{p} / \mathrm{s}$ motion with f/e angle held constant at 4) $30^{\circ}$, 5) $60^{\circ}$, and 6) $90^{\circ}$. The cylinders moved to maintain constant muscle force, thus cylinder travel was equivalent to tendon excursion.

5 Elbow position and cylinder travel was monitored at $40 \mathrm{~Hz}$ and exported via a custom Visual Basic (Microsoft, Redmond, WA) code. Sixth-order polynomial curves were fit to the both the joint angle and tendon excursion data using custom MATLAB software. (The MathWorks, Natick, MA) The moment arm (M.A.) for each muscle was calculated using:

$$
M . A .=\frac{d r}{d \theta}
$$

where $r$ is tendon excursion and $\theta$ is the elbow angle of interest $(\mathrm{f} / \mathrm{e}$ or $\mathrm{p} / \mathrm{s})$. Five trials of each motion were recorded for each specimen. Results were computed for every $5^{\circ}$ of motion and averaged across all trials for each specimen. The standard deviation across

15 all trials of the same type for each specimen was also computed.

\section{Results:}

\section{Measured moment arms:}

Figures 4 and 5 show the results of the measured moment arms for each muscle. Figure 4

20 shows the results for the flexion/extension moment arms of all muscles and all specimens, and Fig. 5 shows the same for pronation/supination. In both figures, each column shows the results of all four muscles for a given specimen. In all plots, the dark 
lines show the averages across all trials, and the shaded regions show the corresponding standard deviations across trials of the same type for each specimen.

\section{Flexion-extension moment arms:}

5 Looking at the biceps moment arm, shown in the first row of Fig. 4, it is notable that the $\mathrm{f} / \mathrm{e}$ moment arm varies with $\mathrm{p} / \mathrm{s}$ angle across all specimens. That is, the peak moment arm occurs at a smaller flexion angle in supination than when in neutral or pronation than when supinated. This observation is implied from the anatomical structure of the biceps. When the forearm pronates and supinates, the biceps tendon wraps around the radius,

10 which alters its effective insertion point. The variability decreases from Specimen $\# 1$ to Specimen \#3. It is interesting to note that the peak moment arms occur within $10^{\circ}$ of each other across all specimens.

Plots of the measured moment arm results for the brachialis are shown in the second row

15 of Fig. 4. As expected based on the anatomy of the triceps muscle, as seen in the third row of Fig. 4, the flexion moment arm changes very little with $\mathrm{p} / \mathrm{s}$ position. As with the biceps, the variability, represented by the standard deviation (the shaded region of the plots) decreases from Specimen \#1 to Specimen \#3.

20 The measured moment arms of the pronator teres display the greatest variability within cases, as shown by the shaded areas in the fourth row of Fig. 4. As with the biceps, the $\mathrm{f} / \mathrm{e}$ moment arm of the pronator teres varies with forearm $\mathrm{p} / \mathrm{s}$ position. It is greatest in pronation and least in supination. 


\section{Pronation-supination moment arms:}

Similar to the f/e moment arms, the biceps $\mathrm{p} / \mathrm{s}$ moment arms show a dependence on $\mathrm{f} / \mathrm{e}$ position, as seen in the top row of Fig. 5. As seen in the second row of Fig. 5, the

5 brachialis $\mathrm{p} / \mathrm{s}$ moment arm is essentially zero for all $\mathrm{f} / \mathrm{e}$ angles.

Similar to the brachialis, it is anticipated that the triceps will have no $\mathrm{p} / \mathrm{s}$ moment arm due to its attachment to the ulna, which remains stationary during $\mathrm{p} / \mathrm{s}$ motion. This is seen in the third row of Fig. 5. The inter-trial variability is more apparent in these figures, and

10 suggests that some slight adjustments to the triceps position were needed to maintain constant force. Small adjustments are not surprising, however, since the biceps and pronator muscles can also produce f/e moments while actuating $\mathrm{p} / \mathrm{s}$ motion. The triceps may have been counteracting such moments. The results from Specimen \#2 do not include the full range of $\mathrm{p} / \mathrm{s}$ motion seen in the other specimens. This is because

15 Specimen \#2 had a smaller range of motion in comparison to the others. The bottom row of Fig. 5 shows the measured p/s moment arms for the pronator teres. There is variation in moment arm magnitude with flexion angle, however, the pattern is less stark.

\section{Discussion:}

A physical aspect of the AGH Elbow Simulator has been validated with a new variation on the tendon-displacement method for moment arm measurement. The measured f/e moment arms vary with $\mathrm{p} / \mathrm{s}$ position for the biceps and pronator muscles, and vice-versa, 
as expected from the way these muscles wrap around the radius during $\mathrm{p} / \mathrm{s}$ motion. Similarly, these same muscles' $\mathrm{p} / \mathrm{s}$ moment arms show dependence on f/e angle, as expected for the same reason. Due to their attachment to the ulna, which remains stationary during $\mathrm{p} / \mathrm{s}$ motion, the $\mathrm{p} / \mathrm{s}$ moment arms of the triceps and brachialis are

5 essentially zero. In general, the variability within cases decreases as the number of specimens tested increases, which suggests that there may be a learning effect of the manual operator actuating the motion. If this variability truly occurred within each specimen, it could represent the viscoelastic effects of the tendon, though that is unlikely given that the same variability is not seen across all specimens. There is no reason to

10 suspect that differences between right and left arm specimens may have influenced the results since the simulator is fully adjustable to accommodate both right and left arms.

\section{Flexion-extension moment arms:}

The measured values of the biceps moment arms fall within the ranges of previously-

15 reported moment arm values. [2,5,12,13,20,21,25-27] It was anticipated that these values would vary with $\mathrm{p} / \mathrm{s}$ position given that the biceps muscle wraps around the radius during $\mathrm{p} / \mathrm{s}$. This changes its effective insertion point and consequently its moment arm.

The f/e moment arms of the brachialis muscle behaved as expected given its anatomical

20 structure. Since it attaches to the ulna and does not interact with the radius during $\mathrm{p} / \mathrm{s}$ motion, the moment arms are not expected do not vary much with $\mathrm{p} / \mathrm{s}$ position. As with the biceps, the measured results here compare well with previously-reported results in the open literature. 
The peak triceps f/e moment arms across specimens are very close in value, though the flexion angle at which they occur is much lower in Specimen \#1 than in the others. This could be due to anatomic variability or due to slight inaccuracies in the calibration of the

5 f/e angle measurement device. Given that the f/e angles for the other muscles measured on this specimen do not differ dramatically from the other specimens, an error in the device calibration is unlikely.

The f/e moment arms of the pronator teres also behave in an intuitive sense given the

10 muscle's anatomy. With an insertion on the lateral side of the radius, the effective insertion of the pronator teres moves proximally as it wraps around the radius in supination, reducing its effective ability to flex the elbow. In general, the variability of the moment arms of the pronator teres is larger than that of the other muscles. This could be due to inconsistent wrapping of the tendon around the radius which would have been

15 possible with the absence of the surrounding tissues. It could also be due to the swiveling action of the pulleys designed to maintain physiologically accurate moment arms. It is possible that, across different trials, the pulleys swiveled at the different elbow angles. Since pulley swiveling is dependent on tendon excursion, a change in the amount of tendon excursion at a given angle which would necessarily affect the computed moment

20 arms. This could be monitored in future work with a rotary potentiometer in the pulley assembly, assuming that there is no slip between the high-strength cables and the pulley surfaces. 


\section{Pronation/supination moment arms:}

The $\mathrm{p} / \mathrm{s}$ moment arms of the biceps muscle vary with $\mathrm{f} / \mathrm{e}$ angle. This is again anticipated because the tendon wraps around the radius during $\mathrm{p} / \mathrm{s}$ motion, which changes its effective insertion point. It is not surprising that the moment arm increases with flexion

5 angle. The variability between trials could be explained by inconsistencies in wrapping during $\mathrm{p} / \mathrm{s}$ motion. With the surrounding muscles removed, the tendon could have wrapped around the radius differently each time.

Given that the brachialis attaches to the ulna, which does not move during $\mathrm{p} / \mathrm{s}$ at a fixed

$10 \mathrm{f} / \mathrm{e}$ angle, it is not surprising that its $\mathrm{p} / \mathrm{s}$ moment arm is approximately zero. This compares well with the previously-reported results. [5,13] The variability within cases (between trials) is quite small as well. Specimen \#2 had a smaller range of feasible motion than the others.

15 The observed variation in pronator teres $\mathrm{p} / \mathrm{s}$ moment arms could be due in part to inconsistent pulley swiveling at different times across trials. The custom pulleys were designed to swivel to maintain physiologically-accurate moment arms but it is possible that friction in the pulley assembly, or inconsistencies in the manual actuation, caused the pulleys to swivel at different $\mathrm{p} / \mathrm{s}$ angles across trials. It is also feasible that this variability

20 is due to differences in the way the tendon wrapped around the radius between trials. Since some of the surrounding tissue was removed, the tendon was less constrained and may have wrapped around the radius differently than it would have in vivo. Similar to the results from the brachialis, the plot from Specimen \#2 appears truncated due to its more limited range of motion. 
This study is one of the few to investigate the dependence of elbow muscle moment arms on both $\mathrm{f} / \mathrm{e}$ and $\mathrm{p} / \mathrm{s}$ position. One of the most extensive previous works to report elbow muscle moment arms was performed by Ettema et al. [12] Their reported moment arm

5 results tend to fall outside the ranges of those reported by other researchers. Several factors could have contributed to their extreme results, including their methodology. The musculotendons were replaced with elastic bands, and required the removal of all surrounding tissue. This tissue would ordinarily influence muscle and tendon paths and could yield moment arms substantially larger than those found with the intact constraints

10 of skin, muscle, and fascia. The precision of the hand-held ruler used to measure the musculotendon lengths could have decreased the precision of their results, though it is unlikely that these errors would cause the dramatic departures from others' reported results. Gardinier and Gonzalez also reported a selection of peak p/s moment arms, but only for the pronator teres and brachialis. [13] Their results indicated that $\mathrm{p} / \mathrm{s}$ moment

15 arms for these muscles change with f/e position, which agrees with the results described here.

A limitation of this work is that a small number of specimens was tested and all specimens were from elderly female donors. While this small sample may not be

20 sufficient to make generalizations about moment arm behavior, it is sufficient to show that the AGH Elbow Simulator can create physiologically accurate moment arms. The results from all specimens exhibit the same trends with respect to the dependence of the biceps and pronator teres on $\mathrm{p} / \mathrm{s}$ position for $\mathrm{f} / \mathrm{e}$ moment arms, and vice versa. Future 
testing should include male specimens, and specimens of a wider age range, to confirm these findings.

The standard deviations, as indicated by the shaded regions on the plots, are sometimes

5 large near the ends, such as that of the triceps f/e moment arms. This behavior is likely due to the end effects of the polynomial fits. Near the ends, the velocity of the movement was necessarily slower, which means that the rate of change in tendon length, or elbow position angle, were lower than during the mid-range of the motion. Thus the limit of the $d \theta$ term heads towards zero, which in turn makes the calculated moment arm approach

10 infinity. Neglecting additional terms at the beginning and the end of the trials could reduce these end effects.

The measured moment arms presented here fall within the ranges of those previouslyreported. $[1,5,13,14,20,25,26]$ This illustrates that physiologically accurate moment arms 15 can be produced with the simulator and demonstrates that the closed-loop force controller offers a practical variation on the traditional tendon-displacement method to measure moment arms. While one measure cannot validate all aspects of the simulator's design, an elbow simulator with physiologically correct moment arms will yield results that are more anatomically and physiologically realistic than can be produced on any other elbow

20 simulator currently in use.

An inherent problem with the tendon-displacement approach for moment arm measurement is the differentiation required by Equation 1. One approach is to use 
numeric differentiation [7] which often requires filters or other smoothing techniques to reduce the noise. Others have taken a curve-fitting approach, fitting a polynomial to the displacement vs. joint angle curve. [5] The curve fitting presented in this work is unique in that polynomial curves are fit to both the tendon displacement and joint angle

5 measurements. The derivatives of each of these curves are computed, and their quotient represents the moment arm, per Equation 1. The polynomial fitting to both the " $\mathrm{r}$ " (tendon excursion) and " $\theta$ " curves permitted analytic computation of the derivative. This technique is not without fault: numeric rounding errors during the evaluation of both polynomials may have introduced noise into the results. However, the averaging across

10 trials, and doing so only every $5^{\circ}$, helped mitigate these effects. Given the larger standard deviation magnitudes near the ends, it seems possible that using even higherorder curves may additionally improve the results. In preliminary work, sixth-order curve fits did not yield an improvement.

15 As with most work, there is the potential to develop future studies based on the results presented here, and room for improvement in the current techniques to offer more accurate and meaningful results. While a more advanced controller (such as PI, or PID) may in theory offer improved force control, the current hardware configuration would produce inferior results due to the increased loop time and corresponding delay in system

20 response. This added delay is a result of increased computation time from the additional gains. The $40 \mathrm{~Hz}$ sampling frequency was the maximum available speed because the ACR 8020 operates in an interpretive manner. A rotary potentiometer could be installed 
to monitor the swiveling action of the pronator teres pulley assembly. This may account

for the intra-specimen variability, particularly for the $\mathrm{p} / \mathrm{s}$ moment arm values.

In conclusion, the moment arm values measured in the AGH Elbow Simulator compare

5 well with those previously-reported in the biomechanics literature. This validates one aspect of the simulator's design and offers insights into the behavior of the moment arms of the elbow.

\section{References:}

101 Amis AA, Dowson D, Wright V. Analysis of elbow forces due to high-speed forearm movements. J Biomech 1980; 13: 825-831.

2 An KN et al. Muscles across the elbow joint: a biomechanical analysis. $J$ Biomech 1981; 14: 659-669.

3 Armstrong AD et al. Rehabilitation of the medial collateral ligament-deficient

15 elbow: an in vitro biomechanical study. J Hand Surg [Am] 2000; 25: 1051-1057.

4 Armstrong AD et al. Single-strand ligament reconstruction of the medial collateral ligament restores valgus elbow stability. J Shoulder Elbow Surg 2002; 11: $65-71$.

5 Bremer AK, Sennwald GR, Favre P, Jacob HA. Moment arms of forearm rotators.

20 Clin Biomech (Bristol, Avon) 2006; 21: 683-691.

6 Buckley MA, Yardley A, Johnson GR, Carus DA. Dynamics of the upper limb during performance of the tasks of everyday living--a review of the current knowledge base. Proc Inst Mech Eng [H] 1996; 210: 241-247.

7 Buford WL, Jr. et al. Muscle balance at the knee-moment arms for the normal

25 knee and the ACL-minus knee. Rehabilitation Engineering, IEEE Transactions on [see also IEEE Trans. on Neural Systems and Rehabilitation] 1997; 5: 367379.

8 Conn J, Wade PA. Injuries of the Elbow: A Ten Year Review. Journal of Trauma 1961; 1: 248.

309 Debski RE et al. A new dynamic testing apparatus to study glenohumeral joint motion. J Biomech 1995; 28: 869-874.

10 Dunning CE et al. Muscle forces and pronation stabilize the lateral ligament deficient elbow. Clin Orthop Relat Res 2001: 118-124.

11 Dunning CE et al. Ligament Stabilizers Against Posterolateral Rotary Instability of the Elbow. Journal of Bone and Joint Surgery 2001; 83-A: 1823-1828. 
12 Ettema GJC, Styles G, Kippers V. The moment arms of 23 muscle segments of the upper limb with varying elbow and forearm positions: Implications for motor control. Human Movement Science 1998; 17: 201-220.

13 Gardinier JD, Gonzalez RV. Pronation-supination moment arms in the human forearm. Summer Bioengineering Conference: Key Biscayne, FL, 2003, pp. 10151016.

14 Gonzalez RV, Hutchins EL, Barr RE, Abraham LD. Development and evaluation of a musculoskeletal model of the elbow joint complex. J Biomech Eng 1996; 118: $32-40$.

1015 Hollister AM, Gellman H, Waters RL. The relationship of the interosseous membrane to the axis of rotation of the forearm. Clin Orthop Relat Res 1994: 272-276.

16 Johnson GW. A Follow-Up of One Hundred Cases of Fracture of the Head of the Radius with a Review of the Literature. Ulster Medicine Journal 1952; 31: 51-56.

1517 Johnson JA et al. Simulation of elbow and forearm motion in vitro using a load controlled testing apparatus. $J$ Biomech 2000; 33: 635-639.

18 King GJ et al. Single-strand reconstruction of the lateral ulnar collateral ligament restores varus and posterolateral rotatory stability of the elbow. J Shoulder Elbow Surg 2002; 11: 60-64.

2019 King GJ et al. Metallic radial head arthroplasty improves valgus stability of the elbow. Clin Orthop Relat Res 1999: 114-125.

20 Lemay MA, Crago PE. A dynamic model for simulating movements of the elbow, forearm, an wrist. $J$ Biomech 1996; 29: 1319-1330.

21 Magnusen JP. Design and Fabrication of an Elbow Motion Simulator. Mechanical Engineering. University of Pittsburgh: Pittsburgh, PA, 2004.

22 Miller MC et al. The effect of component placement on knee kinetics after arthroplasty with an unconstrained prosthesis. J Orthop Res 2001; 19: 614-620.

23 Morrey BF. Radial head fracture. In: The elbow and its disorders. W.B. Saunders: Philadelphia, 2000, pp. 341-364.

3024 Morrey BF, Askew LJ, Chao EY. A biomechanical study of normal functional elbow motion. J Bone Joint Surg Am 1981; 63: 872-877.

25 Murray WM, Buchanan TS, Delp SL. Scaling of peak moment arms of elbow muscles with upper extremity bone dimensions. J Biomech 2002; 35: 19-26.

26 Murray WM, Delp SL, Buchanan TS. Variation of muscle moment arms with elbow and forearm position. $J$ Biomech 1995; 28: 513-525.

27 Pigeon P, Yahia L, Feldman AG. Moment arms and lengths of human upper limb muscles as functions of joint angles. J Biomech 1996; 29: 1365-1370.

28 Seireg A, Arkivar R. Biomechanical analysis of the musculoskeletal structure for medicine and sports. Hemisphere Pub. Corp.: New York, 1989.

4029 Sharkey NA, Hamel AJ. A dynamic cadaver model of the stance phase of gait: performance characteristics and kinetic validation. Clin Biomech (Bristol, Avon) 1998; 13: 420-433.

30 Werner FW et al. Wrist joint motion simulator. J Orthop Res 1996; 14: 639-646. 

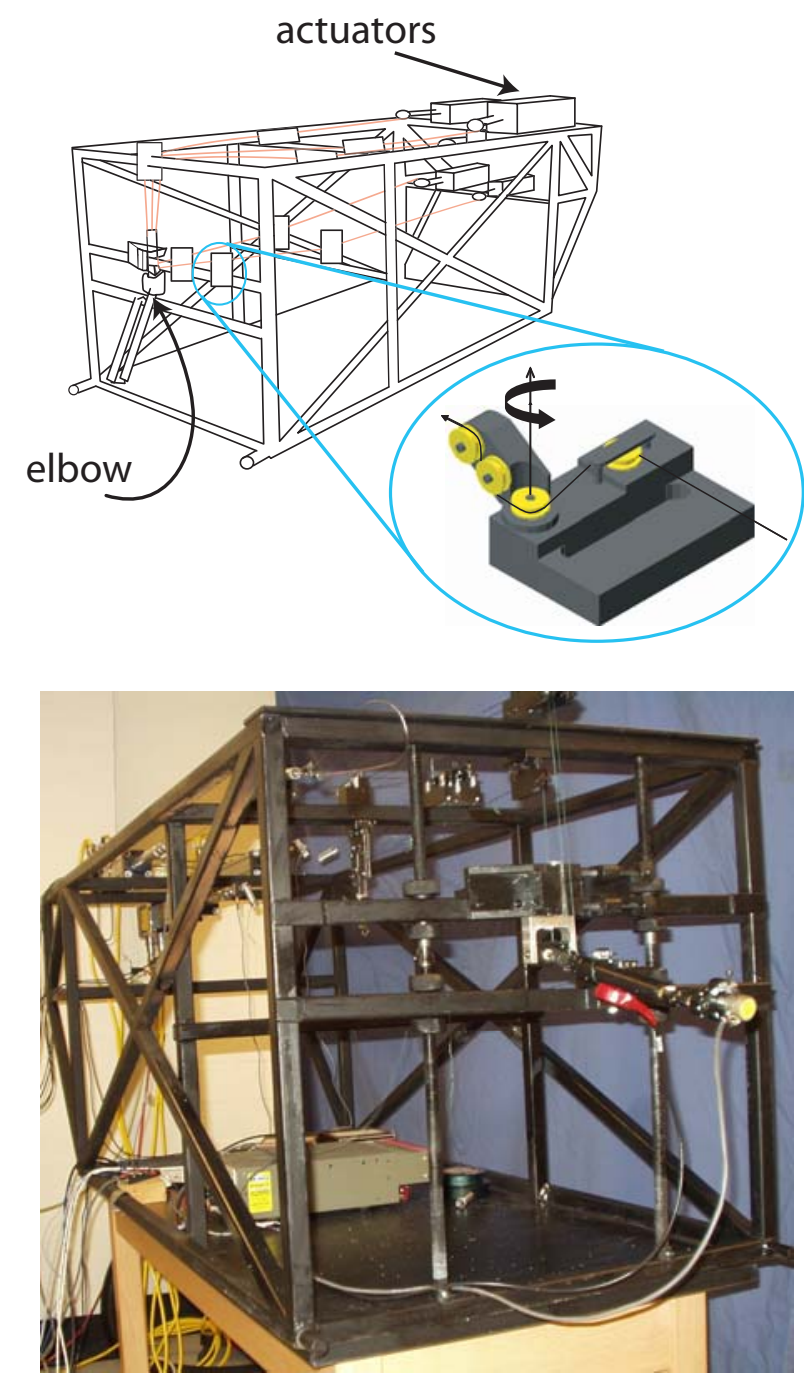

Fig. 1: Drawing and photo of AGH Elbow Simulator. The inset in the drawing shows a custom pulley assembly. 


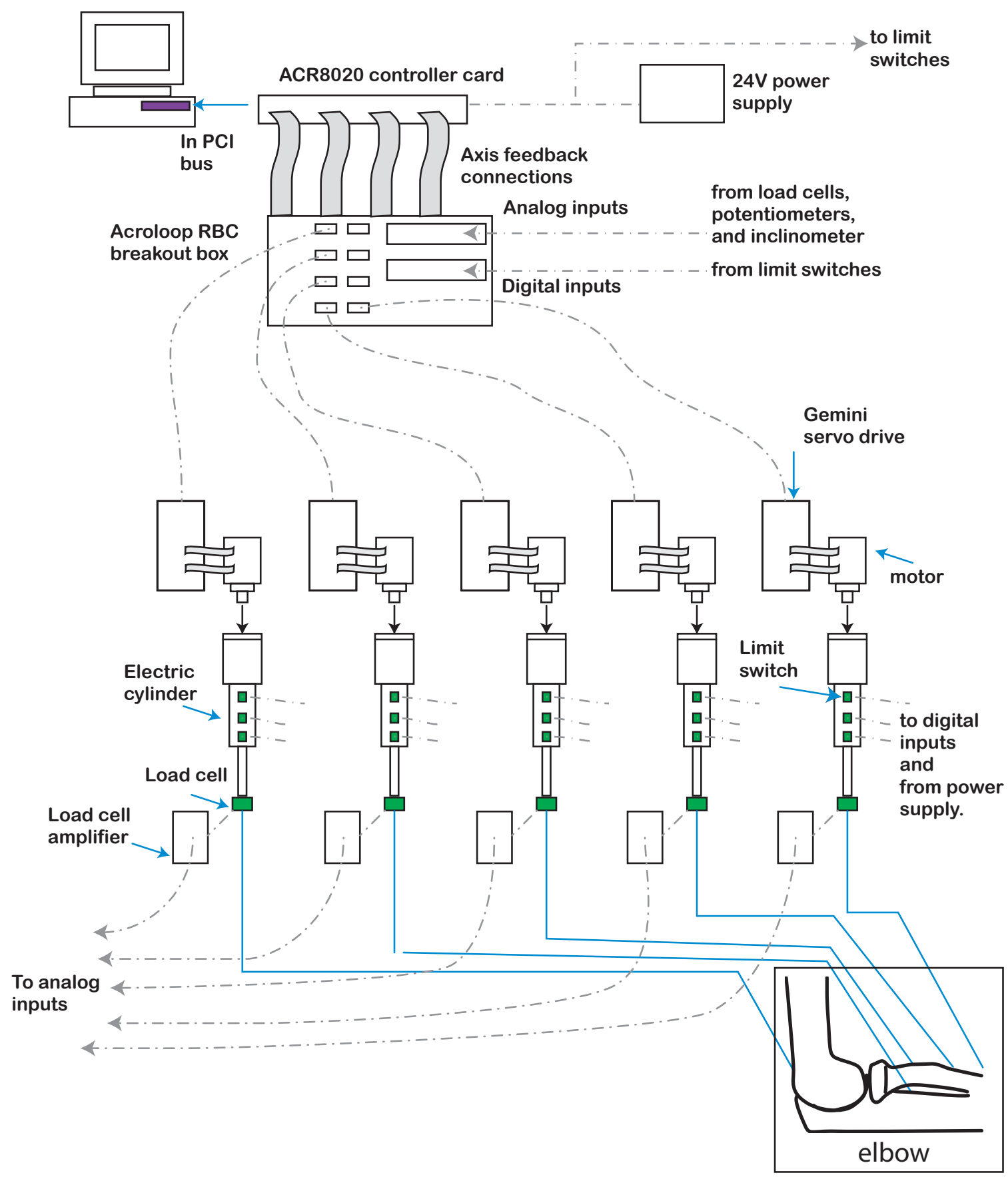

Fig. 2: Overall schematic of simulator hardware. 


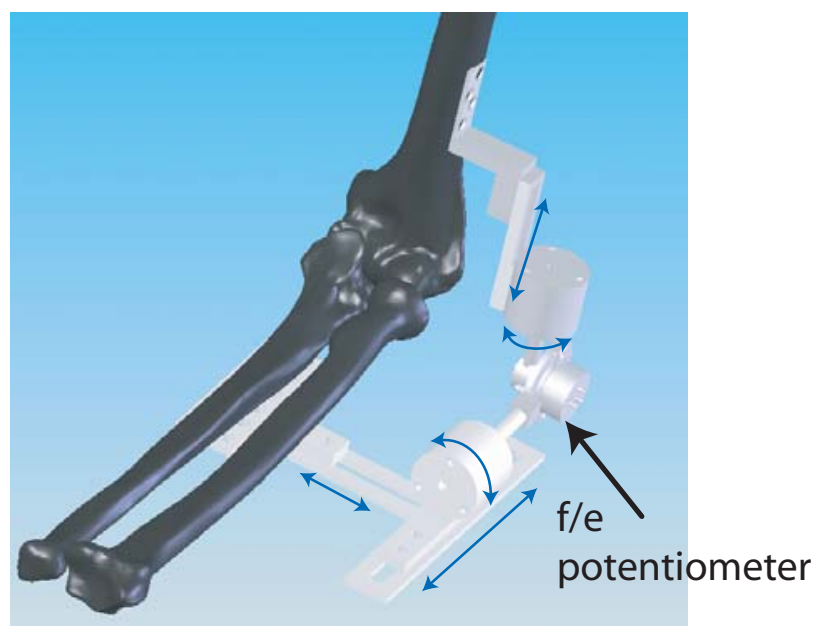

(a)

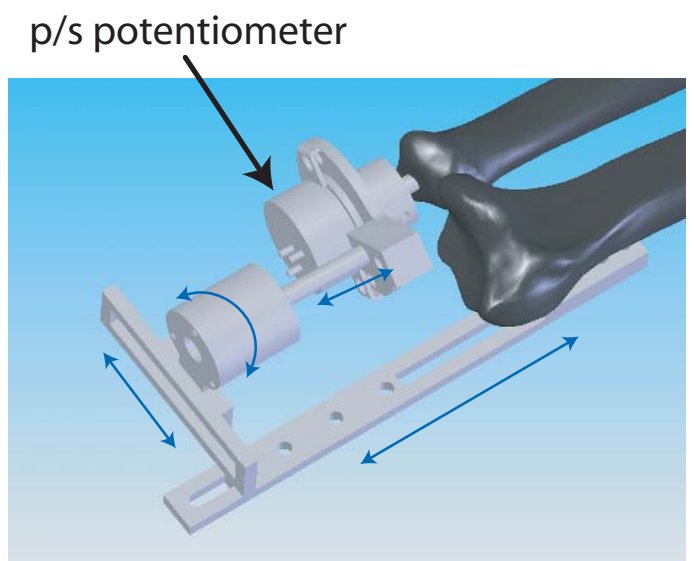

(b)

Fig. 3: Custom potentiometer mounts to measure f/e and p/s. 

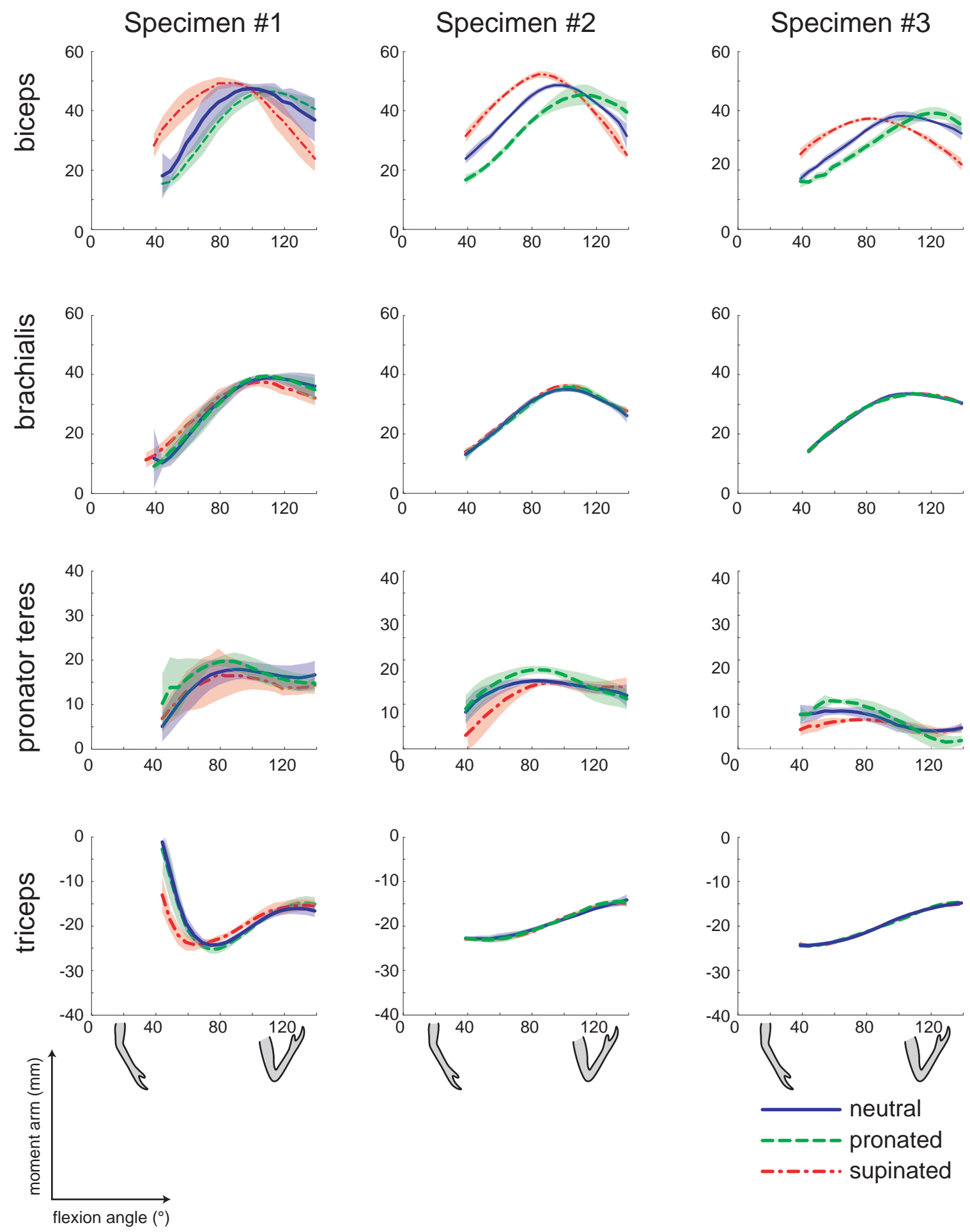

Fig. 4: Flexion/extension moment arms for all specimens. 

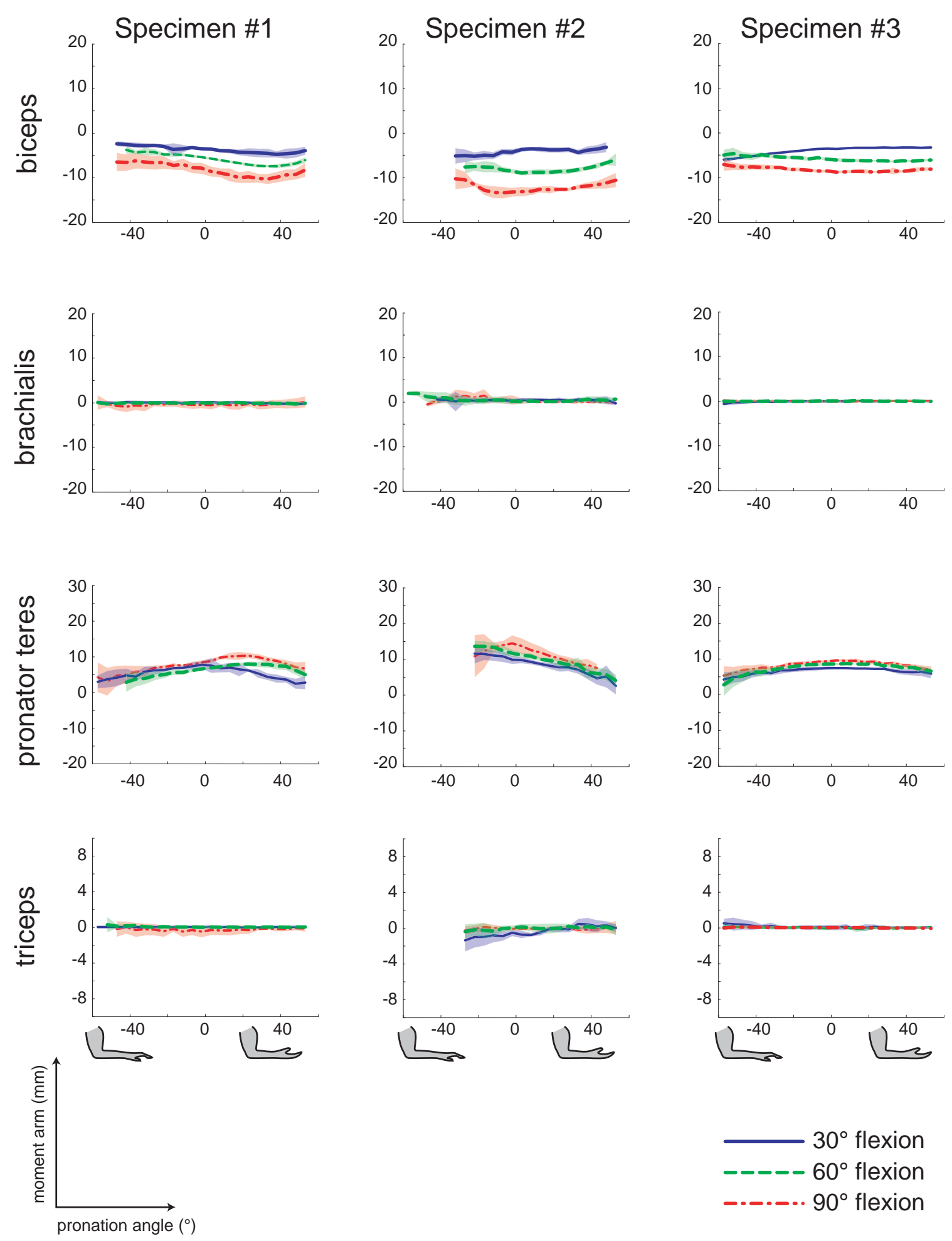

Fig. 5: Pronation/supination moment arms for all specimens. 